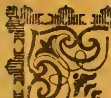

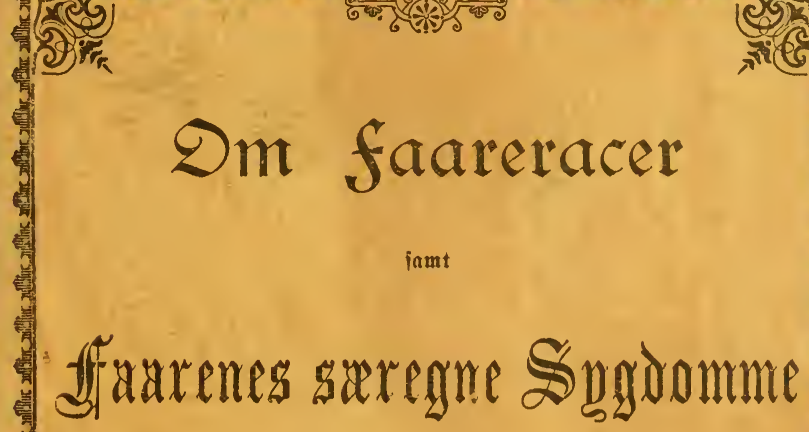

Bg Deres Beffanding.

Mred et Unhang om Slagtning.

$\mathfrak{4}$

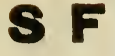

968

G25

톅 (2) (5) d5. (6) a f $\mathfrak{4} \mathfrak{n} \mathfrak{g}$,

Norit=amerifanff Dyrlage.

Cbic ago.

Stanoinaven's Boghanocl.

1887. 


\section{LIBRARY OF CONGREESS,}

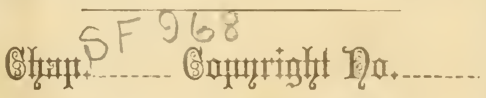

$$
\text { Shelf } \div 25
$$

UNITED STATES OF AMERICA. 


\section{Oill Jaareracer}

jamt

\section{fHarencs sirterne Sugdomme}

- og deres Bachanding.

2ned et 2lnhana om Slagtuna.

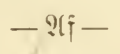

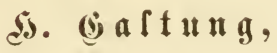

Morifsmmerifnuit Iurlmge.
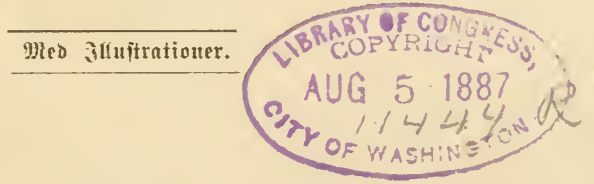

Cbicago.

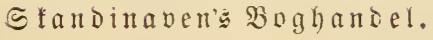

1887. 


\section{SF 968 \\ .625}

Entered according to Act of Congress, in the year ISS 7 , by

John Axderson \& Co.

In the Office of the Librarian of Congress, at Washington, D. C.

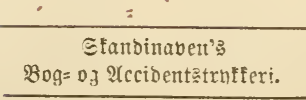




\section{forord.}

Ia ber $\mathrm{i}$ ben morif-anterifanje Siteratur iffe gistif har waret noget jelvitenoigt og prisbilligt Efrift, ber ipecielt bax behandet bette Emute, maa bet antages, at her ex Irang tiljtede for et jandant Strift, og Dnjet

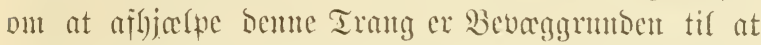

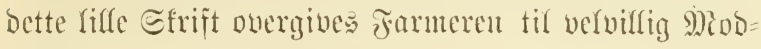
tagelic.

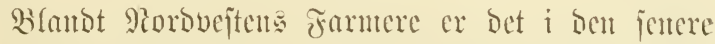
Tib blevet en ledende Tanfe at ofre Faareavlen nere

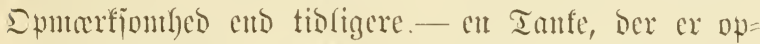
ftanet formedeljt ben Sijendagjerning, at Svincavlen, ber tioligere nogjorbe en iffe mexientlig Jnotergt for Farmeren, i ben jenere Tib hax vijt jig mutore foumento, bels forbi, at Sumefoleraen, der funes at trobje alle jorebnggeljesmider, optraber mere og mexe herjente, og bels forbi, at Priferne, grumbet paa frere curoperiffe

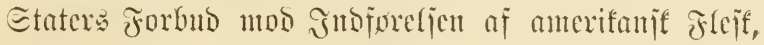

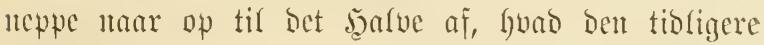
gioride.

Ia ber tillige ex alfe Iegu tir, at lloforjelen af Elagtefaar til Europa $i$ en nar Frentis vil tage et iteoje jtigento Dpjuing, ligger ogja heri al spepororing til at bentte jig af jammes Forbele og ofre Sagen ben sinnerfombed, fom ben fortjencr.

\section{Eัorfatteren.}





\section{(1) fat fareracer.}

Iet forite Sporgsmaal, Farmeren opfajter, naar han: Bejtemmelje er tagen om at ville holde Faar, er: Guilfe Racer maatte pasje bedit for be forbaan= benvarende lofale Forfold, og fum me itorit llofigt tir Fordel underforbes. Det er eit Riends= gjerning, at her er en Mangde Faareracer, Arter

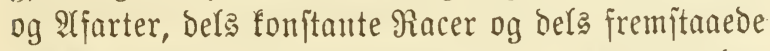
ved firybning. Paa mange Steder i Europa lyar man ofret Faareablen at buffelig spmorfiombed, og Bland de Rande, fom i benne Fetning ftaar $i$

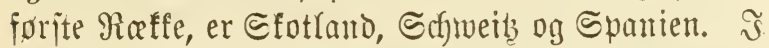
lange 2larraffer har ent inftematiff Dporat og $\mathfrak{B e}=$ Gandling i Disje Rande bleven Faareavlen til Iel, og

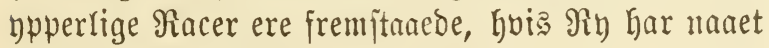
til de fiermejte Egne.

Faareavl ex en nteget gammel Naringavej, der gaar tilbage lige til be gammel=teitamentrige Dage. Dlotidens Folfellag, der for det mefte var গomade= itammer, inntes at Gave jat fin ?are $i$ at have det mefte og bedite forg, idet dent patriarfaljfe 9)inn= bighed vajentlig bejtemmedes efter bette. Der var 
Dog neppe i bisfe Tiber mange Faareracer, frem=

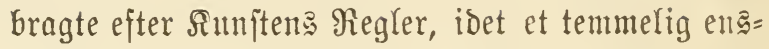
formigt og rigefremt Frembringelfesjet har gjort fig gixldende, og bette har fin Forflaringagruno deri, at de lofale og llimatiff Forholde iffe frem= bod faa ftore Uregelmasjigheder fom jenere, da dišfe Dnr bleb ipredte fra den ene sordens Ende til Den andent.

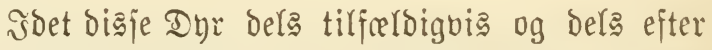
Beregning fpredtes rundt $i$ de forffjellige sande unber be mejt forffjerligartede rofale og flimatijfe Forbold, er det en Selviplge, at de \$rag og Frem= tredeljesformer, fom de bejad i saturtirfanden, mere og mere tabtes og antog farafter efter De fted= rige Forfold, fom de tilfaldiguis bleve henjatte under. Et Dyrs Sirundormer fan unber Ratur= tiljtanden bibefoldes uforandret, faalenge fom det befinder fig inden benne, men aldorig faajuart bliver denne forrnffet, forend en iojuefaldende Forifjel fra bet oprinderige fan fpores.

I vild Tilftand har Faaret det tilfelles med de flejte andre Dyr, at Det har et grovere Udjeende. For at fum moditan Bejrrigets ffiftende Birfuin= ger er bette ogjaa nobvendigt, og Naturen forger Da felv for at bibringe det den formodne Saaroforfed. urben er ba langt ftivere og grovere, og Ben= og Mrujfelbyguingen ftorf og harmoniff noviflet. Disje Egenffaber tabes alt mere og mere $i$ jamme Forfold, fom det moer Mennejfets Serreopmme 
Griver tir Del en Behandring, der mere og mere af= viger fra Naturtilftanden og de frie Snftutters Brug.

Det var forft ejterat Faareablen blev SSjenjtano for en orduet Saringsgren, at Raceforbebring bed Srydsning blev en Mlulighed. Enfelte Faareboldere fororede nemlig helft Dyr, ber havde en ftert fjod= producerende Fone, atter andre lagbe ndeluffende an paa, at jrembringe megen og goo urd, medens atter andre lagoe Iyngoepunftet par at je begge disje Egenjfaber nopragede $i$ en og famme Race. Iet, fom atter igien var bejtemmende for denne Indode= ling, var de lofale og flimatiffe Forhoro, . Der fom ell Folge af $\mathfrak{a p b v e n d i g h e d ~ m a a t t e ~ r a a b l p o r g e s . ~}$

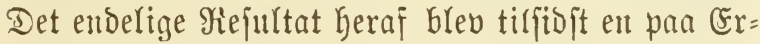
faring $\mathrm{Dg}$ Sidenffab planlagt Fremgangamaade, foorved de ftorite Fordele med mindit mulige $110=$ gifter funde vindes, og de flefte Seinoringer for et velordnet og lonnende Faareaulinitem undgaaes.

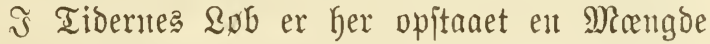
2irter og arfarter af Faar, ibet hoer af bisje har antaget Proeg af ben Belyandingsmaabe og be $l_{0}=$ fale og flimatiffe Forholo, mber huilfe de har voret ftillede. Beb formuftig og omjorgafuld sndaul, det vil fige, ndon Blanding med indoforte og ganfe af= vigende Racer, har bet Inffedes at fremitille $u \delta=$ mrerfede Arter, Der befioder alle onjfelige Egenjfaber for dette eller hint Djemed. Biftnof ma det er= fiendes, at ber mange Gange, jaavel ved sndoul fom 
ved Rrydsuting med antore Racer, er begaaet ftore Jejltagelfer, grumbet paa manglende Indfigt $i$ at

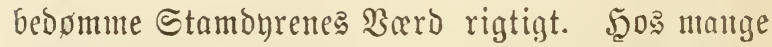
er benne Ridenjfab, der ytrer fig $i$ planlos sage:

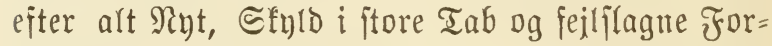
haabninger, ment Frfaring har og vil frembeles blive den Rettefnor, fom bor befolges, og fuorved Fejltageljer unogaaes.

Siryosning er oog ofte en anbejalelfesvordig Ting, da en Races Egenjfaber tiot bed fortjatte uheldige Dmitænoigheder fall tabes, og ba giorlder bet iffe alene at frembringe de gobe men endog langt ablere Egenffaber, eno Stamoyrente af famme Face bejad, alt grumbet paa en formuftig Dporat og \$rleje

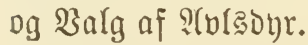

Bland de mange Racer af Faar, fom $i$ broget Blanding fan jes runot $i$ be norboejtlige Stater, ment for de fleites Bebfommende dog mejt $i \varsigma_{y} \delta=$ veiten og Syoditen, maa næunes jom de bxjentlig= ite: Merinofaaret, Reicefterfaaret, Sotsworbfaaret, Rincolnfaaret, Sugjex= Dounfaaret og Cheviot faaret.

Merinofaaret giælber for at bære Berbens mejt finuldode Faar, og llloen anvendes blot $i$ de fineite Alæbesfabriffer. Meñ mau maa lxage Marfe til, at Denne Sूovedegenffab tabes hos Merinofaaret $i$ famme Srab, fom lofale og flimatiffe Forbord iffe itemmer Doerents mes Dets Forbringer. Det er nem= lig bein mejt fjalne Faarerace, man har, og fantun med Fordel underfoldes paa Steder med et Rlimat, 


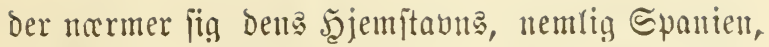

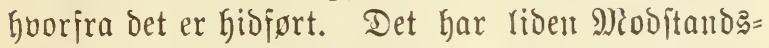
fraft ligeoverfor folot Beirlig, og bliver bet ento med Tioen gientem flere Slaggtled lofafiferet, jaa fall man vore vis paa, at Dets Sovedegenffaber for ell ftor Del er tabte, idet dent fine $\mathrm{u}$ ro er vegen Blabjen for ell grovere.

Qeiceiterfaaret er af engelif Dprindelfe og er en fonftant Race, frembragt bed Rryosning af gooe Ifvlabyr. De er af enl langt itorre Segemabugnting ent Merinofaaret og underfoldes meft for Sijobets. Sfyld. Engelifmerndene ere nemlig ftorfe Faare fipoipijere, medens ?tmerifanterne hibtil iffe har efterfigntet fine Stammefrender i bente Sag, men Tiben vil nof ogiaa heri bringe ent Foralloring. Baa Steder, yoor Faarefipo er ell efterfpurgt Bare, vil det viftnof under ellers gunjtige $\$$ mitrubigheder, loune fig at underforde bem. Mlen man ma iffe labe noe af Sigte at froe bem goot, ibet be maa have rigelig Fobring og fraftige (Sircesgange, uoen lyoiffe de iffe giver forouffet Uldbytte. For Norbvejtens

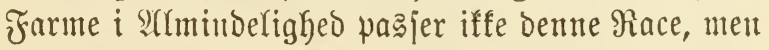
giver, fom ovenfor newnt, goot Hobntte, hoor ben finder tiljitreffelige Betingeljer for fin Trinjer.

Cotatwoldfaaret er ogjaa en ftor Faareruce, oer fornemmelig ubmarker fig ved fute hoje Ben og hojt= ftillede Shoved. Sgjaa dette er af entgelif Sprintoelfe, og er elt fonitant Race. Det jtan i hoj Ânjeelje paa Steder, hoor Det tribes goot, ment heller iffe 
Denne Faarerace pasjer for Porbvejten, idet Den ftiller altfor ftore Fororinger til be flimatijfe og dixtiffe Forbold. Det egiter fig bedit for ftorre Fraarefarme i Sybueften og Syden. (3jientem Int)= abl er Det blevet betydelig forbebret og ffal lomme fig goot paa Steder, ber i naturlig Sूenjeende pasjer for Det.

Cot3moldracen er $i$ Størrelje nait den forbe= brebe Rincolnrace, men ogja det fioftneronte pasjer bebit for Untorfyold i ftore Floffe eller paa fraare= farme. Lincolnfaaret or $i$ tropąlig Şenfeende det ftorjte, man har her i Landet, og har ent ualminde= lig lang og glindiende lll, ber mobertiben gives Nannet Lustre Wool.

Susferc=Dotwmacent er en $\mathfrak{A}$ rt fortulbet Faar og jfal bare Landets jmuffefte Faarerace huad Hojeen= Det angaar, men pasjer rigejaaliot jom det fornerbnte for Rorbvejten. Det har tiltruffet fig ftor $D p=$ marfiombed paa mange Steder og har fyoligere en iffe riden udbrederfe. Medens f. Ex. Cheviotfaaret uomerfer fig ved aldeles uldfrit eller "jnaut" 5ूoved og fnane Ben, er Sugjer= Downracen mere ulorig overalt paa disje Legemsoele og er tildels jtorre eno bet foritnconte.

Den Faarerace, fom bedit af alle turbe egue fig for Norobejten, er Den jafaldote "CGyeviotrace." Dent er af Midoelftorrelfe, er fraftig uoviflet, med mid= déls goot urlobelorg og er af ifotit Dprindelje. Det er en fouftant Race og er elt af be mejt fulofomme, 
man har, idet be er letiobrede og itcerfe rigeover= for verlende flimatije Forhold. Dentue Race for ener $i$ fig begge de Egenffaber, fom Rorbveftitaterne forbrer, nemlig $\mathfrak{H L D}=\mathrm{og}$ Sijobrigoom. Rigelebes nomarfer ben fig ogiaa ved ftor Frugtbarked noget, fom iffe fan figes om alle Faareracer.

Bed en rigelig Fooring og forburigt god $\mathfrak{P}$ reje formeres bente Race ualmindelig lurtigt, og Dens Sxrprag fines at fremtrobe $i$ Defto colere Sfif = felfe for hvert fommende Slagtled. Man bor iffe opblande den med andre Racer, ment nobes mant til at opfriffe ben for at mogaa for ner Slægtifabs= avr, bor man forifaffe fig Şandor fra andre $\mathfrak{S}$ jorber af famme Race.

Stambyrente bor mindit vere et oy et halvt (ar gamle, forend de anvenbes til SIUl, ba man fra Dor af yngre arroer iffe fan vente et fraftigt og goot $\mathfrak{Q}(\mathfrak{f}=$ fom. De maa ubuxiges med $5 m$ hu, da dette $i$ hoj Brab betinger et fraftigt og fund affom. Uro= masjen bor bore rell og ellsartet og maa beftaa af rene Stablers, jo mere frufede defto beore. Maif lerue maa vare vel ubviflede, Sovedet af Midoel=

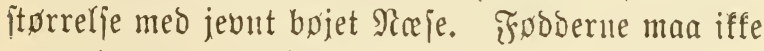
bore lange, ment fraftige og rette. Sryojet hos Salnogret maa iffe vore breot og hojt, famt uben Frejl, fremfaldot ved urnffestilforlde eller paa andent Maabe. Pveret bor vore rmbagtigt med \$atterne (Gponerne) ftiflede vidt fra huerandre. Brbftfasjent 
bor vare fremitaande og bred mellem Forbenene. Stamonrene brr vare af lige Storrelfe.

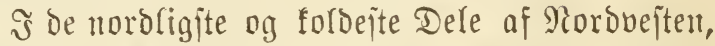
hyor der er $\mathfrak{A}$ luledning til Udegang $\mathfrak{Z}$ interen over, $f$.

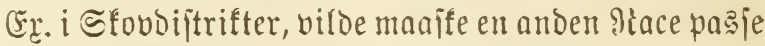
goot, memlig dell faafalote Blacffacerace. Den er under Midodiftorrelje, og, jom ?avuet antyder, fort i Sovedet og har jortipattede Foover. Denne Race er temmelig haardjor og afgiver ganje rigeligt Ripo.

Sfotterne or her i Randet ubetinget de bebite Fanceulere, og be, ber maatte tante paa at brive Faareavl, burbe henvende fig til Slotlanbere for at fjobe ?ulsonr, ba ingen ?ation her har beore Sfjou paa jamme Sag end de.

Det figer fig jelv, at Mlsungen af de Faar, man agter at mberforbe, ftrengt maa rette fig ejter be mere eller mindre gunjtige forfold, unber boilfe man er fillet.

II.

faarets hpppigite Spgoomme fant deres - Behantioling.

\section{Solif.}

Naar Faaret har utilitreffelig 2 ogang til Band eller liber af Feberingoomme, angribes bet tiot af Solif, Der bels ogfaa fremfaldes veo, at man fajter 
Salt til dem paa jandig Srrund, og at det unber Sptagelfen af janme tillige fluger ent Der Sant, Der bringer Foritnrrelje i Fordojelfesorganerne. Engoommens Şeftighed beror paa De STarjager, fom har fremfardt den.

Sjendetegu.

Dyret vifer ftor Uro, tuinger Scaren opad og bojer Bagbenene, idet det trienger paa fom ved Fobjelapeer. Hnder de ftorite Smerter Irgger og reijer bet fig unflabelig og inbtager $\mathrm{i}$ \iggente Stil= ling en vuggende Bevagelie, medens det fteoje jer bag no med et mobfarbent Blif.

\section{B̉e ha lld}

Denne maa ydes faa hurtig fom mulig, da Faa= ret er et af be Dur, fout hurtigit brebes af bente Eygon. En liden SInfterpprojte maa i bette Til= forlde anventes, og flimede firtifterer firtes flere Sanalige.

Snllowortes giver mall:

Castor oil................. I I Ounces.

Glauber's-salt, powd............. I Ounce.

Mix.

En faaban Portion gives med tre Timers Mel= lemrum, og et $\mathfrak{P a r}$ Portioner plejer i ?llmindelig= hed at vare tirftroffelig. 


\section{Diarrboe.}

Demne Sygoom angriber ofte Fuarene i Şojt $=$ og Baarmanneberme og er tiot af ell farlig Patmr, idet den dels optraber med jmitiom siarafter.

\section{Rjendetegn.}

Der ffer hyppige Udotommerjer af flydende, Dels ildelugtende Erfrementer, Der flaber fig fait til Scalent og Bagbenente, hoorved et Diarrfoejugt Int fan jees paa lang affitand. 9) Mab og Driffelyften er i jlemme Tirferbe betydelig formindffet, og en giennenganenbe Slaphed fpores hos Drret, idet bet foretreffer at vanore omfring alene. Sræfterne og Sूulbet aftager

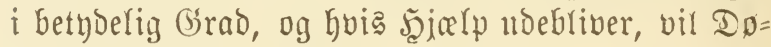
ben indfinde jig.

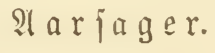

Disje fan vore forjfjellige, og Deriblanot er For fiolelje den hyppigite, idet f. Fr. ftrax efter Silp= uingen Dyret fan uojextes for foldt og regnfuldt Beir. Rigeledes ved baarligt Foder eller af Şavne= gangen, hyor Dyret har ipijt visje \$lanter, der iffe ftemmer med Fordpieljens Farbringer, eller det fan vare ell arvelig Sygrom, ber periodevis giver fig tilfjende.

$$
\text { Be } \mathfrak{a} \mathfrak{n} \text { ori } \mathfrak{i} \mathfrak{g} \text {. }
$$

Man maa underioge, huad fom fan vare ?ar= jagen til næunte Sngoom, og nof̈nbes denne, foger man at mogaa eller fjerne belr. Fooret maa beftaa 
af fajte og Gindende Stoffer, jaajom haffet Jूalm i lige Blanding eller om Sommeren med vel= torret Љุo.

๔om indoortes 9) Midrer anbefales:

Green vitriol ............... I Drachm.

Bark of oak, powd............ I Drachm.

Root of gentian............... Drachms.

\section{Mix.}

En jaaban Portion gives to Sange Daglig, indil Beoring jpores. De af llotommelferne tilféfiptrede Steder, janjom Saale og Bagben, renjes, idet 11 roen flippes bort. Er det untigt bor bet eller be inge Dyr afjonores fra de friffe for at forebygge Murig= heden af at overiore ๔ugoommen ved Smitte.

\section{Drcicinge (Arimgijufa).}

Bibenjfaben laar gootgjort, at Aarjagen til benne Sygoom er ell Bloreorm, der, ved at novilles $i$ Şjernejubftanjen, bringer Forjtyrrerfe $i$ dennes Funftioner, faa at গervevirfjombeden flappes. Un= Der et fterft Forftyrrelfesglą vifer den fig fom en lebet Masfe med mange Şoveder, der ere $i$ en vibre= rende Bevagelfe. Forent Blareormen opdagedes, troede man, at paajialdende Sngoom var Sjerne= betanderle.

凡jendetegn.

Man marfer hos Dyret forit en paafaldende Slovked med Tilbpjelighed til Sajtninger med S్নD= 
vebet, forft i ubeitemte Retninger, fenere mere til ben ene Side end den anden. Layger f. (5r. Dnret Scovedet mere tir hojre Side, bevijer bette, at $\mathfrak{B l a r e}=$ ormens Sperationer foregaar paa Sjernemasjens hojre Side. Tilfidit Gegnnder Dyret at trabe ent formelig Rutboands, altio til en beftemt Side, og Det er efter bisje Bebxgerfer, Sygoommen har faaet Ravit.

\section{Bebanding.}

Det er iffe altio, man fan ubrette noget ved $\mathfrak{B} e=$ handingen, ffiont bet $\mathfrak{i}$ enfelte $\mathfrak{T}$ ilfalde nof fan $\mathfrak{l y} \mathfrak{f}^{*}=$ fes; naar heldige Dmitandigheder trabe til.

Sarelabning er Det nuefte og fifrefte M) Ribdel, og dent fau ndofores ved at tage en $i$ Forlyord til Tyrets Storrelje afpasjet M) og famtioig indimore ßanden med Terpentinolie. Under enfyer Dmitandighed vil Blaredrmens $110=$ vifling hemmes og i heldigite Tilfalde forityrres.

\section{Stivframpe.}

Dette er en farlig Sygoom, der forbrer al $5 p=$ mærffombed, hvis Ruren ffal funne faa et heldigt Udofard, da Gfterladentyed ofteft har Doden til Folge.

$$
\mathfrak{R} \text { jentetegn. }
$$

- Man= nicerfer forjt en vorende Stiohed og Ban= ffelighed $i$ at bevage jig, idet faavel Bsanglemmerne fom ifar Salfen junes at have tabt fin Bevagelig= 
hed, og det funes ogjaa, fom om disje Regemsole er tyffere ent under normale Dmitandigheder. Dptrreder Sngoommen dodelig, taber Dyret tiffidit Ennen til at bevage fig. Mlablyiten tabes, Dinene ftirrer fift hen for fig og Forbenente traffes ino under Bugen. Tiffidit falder Dyret overende og fan iffe mere reije fig. Er Doret jamtioig hermed ubjat for forst eller regufuldot Bejr, er Stillingen defto farligere.

$$
\mathfrak{A} \mathfrak{a}\{\mathfrak{a} g \text { er. }
$$

Som tif jaa mange anbre Sygoonme er For $=$ fiplelje ogiaa her buppigt $\mathfrak{A}$ (arjagen, ibet Dyret iffe er blevet tiljtraffelig tilfeet ftrar efter ßlipiningen.

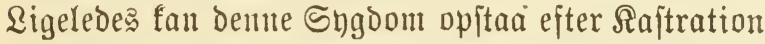
af Bredere, ifar huis demte er nofort under uheldige tlimatiffe Forbold eller af elt urbet Dperatør.

\section{behand}

Dyret bør abjfilles fra de porige og, om muligt,

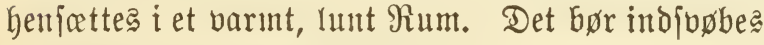
$i$ et Ixppe eller lignende.

эnovortes giver man:

Nux vomica................ I Drachm.

Root of valerian ............... 2 Drachms.

Mix

En faaban \$ortion gives Dyret to Bsange i 24 Timer. Samtibig fant man prove at labe det faa Ciot lumfen Meldriffe, boori liot Salt, og ellers lade det have $\Re$.

om zaareracest. 2. 
Sois bet inden otte Dages Forlob fines at vare rivligere, vil Selbredelje paafolge.

\section{9yerbetandelic.}

Denne Sngoom angriber tiot 9loberbnetheftigt eller remfurldigt i Forhold til 2larjagerne. Under enfyer Dmitentighed maa man have $D_{p} p=$ marfjombeden itide hentent paa Sndet, jaameget mere fom det i armindelighed anyriber de bedite arulabyr.

$$
\text { Rjendetegn. }
$$

Voeret er ftcerft opjoulmet, haarot og varmt. Er ङngoommen af det brandende Slaģ, har शyeret et blaajort ubjeende, der tnder paa en forbojet Blobtilftromning til famme. Melfen ophorer og $i$ dens Sted fan man udmelfe en jeig, flabrig $\mathfrak{B} \propto$ dife, der tildels er blanbet med Bloditrimer og har en egen fur \&ugt.

$$
\text { A arjager. }
$$

Den vigtigfte Uarjag er i Armindeligheo For: fjølelfe ftrax efter Lamuingen, eller at Lammet paa en eller anden Maade fommer afoage, og Melfen fáaledes iffe regelmosfig efler flet iffe ubtommes af Yveret; rigeledes soulft i famme, fremfaldt ved yore Bold; famt Utrenlighed i Blodet.

$$
\text { Be } \mathfrak{h} \text { a d } \text { I } \mathrm{ing} \text {. }
$$

Yveret maa nomelfes ofte, eller man laber flere Lam ffiftevis uopatte bet, forfanviot Mlelfen iffe er 
bleben uren, i hoilfet Tilforlde det maa nomelfes med 5ূaanden. Saalenge Yveret iffe foles haarbt og forbardet, fan mant to Bange baglig paabade det et lunfeut, tuft 2rffog af Buck-besn, men gois פyeret foles haarot, jpentot og uden bejentlig Barme, anbendes folgende:

Iodine ...................... . Drachms. Camphor ................... D Drachms. Lard $\ldots \ldots \ldots \ldots \ldots \ldots \ldots, 4$ Ounces.

\section{Mix.}

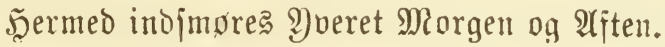

Эnlowortes giver man:

Glauber's-salt................ I Ounce.

Saltpetre................... I Drachm.

Mix.

Dette oplojes i $\mathfrak{B a n d}$ og en faadan Portion gives to Bange Daglig, indtil Beoring ffer. Naar Melfent er bleven ren, fan Lammet atter patte.

\section{Efab.}

Dette er en 5ूubingoom, jom angriber alfe Faare racer, fajpturt Betingeljerne for bens Trivjel er tilftede. Det er ell, om ello iffe abjolut opoelig, oog. meget flem Sygoom, ba den i hpjefte Jsrad er jmit= fom, og Ejeren bil bline paafort betndelige $\mathfrak{T a b}$, om Dett angriber ent hel Faareflof. Dens hurtigit mu= rige Udorbodelje er jaaledes bet fifrejte Miobel mod.

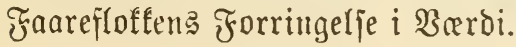


Rjendetegn.

Diaje er forjt en ftorl Silpe, idet Dorene jfub= ber fị mod enfoer Ssjenftand, be fan fomme nar, Goorbed Ulden afgnaves og ber opitnar faare, af= gnavede ßletter, fom overtroffes med Sforper og Sfurv, under hoilfe ber findes Saar, indeholdende en flabrig Materie. Den Mlo, Der iffe ffubbes af, fammenvifles, faa at Dyret faar et hojit uhyggeligt ubjeende. Efabet begynder alminderig paa Saljen

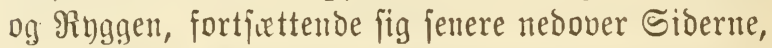
og ved at befole Dnrene fan man danne fig et $\mathfrak{B} e=$ greb om Elabets Ubbrebelfe og Birfninger. Dy= rente afmagres $\mathrm{og}$, hois iffe en formuftig Bebanbling itide intraber, vil flere af Dyrente Do fom Folge af utrivelighed og aidelfer.

\section{Aarjagen}

er fra forft af en baarlig Behandring, faajom baarligt Foder og ubeffnttede Tilfoldafteder. Sentere ffer Det-gjennem ऽmitte, og hyert enejte Dyr inden $\mathfrak{F} l o f=$ feir vil da blive angrebet, og $i$ Sarbeleshed er magre Faar Gjientand for Sfabets Godttyffe.

\section{Beha d d $\mathfrak{i} \| \mathrm{g}$.}

Ta det er et levende, mifroffopiff Dyr, jom fal des Sfabmiboen, der foraarjager Sfabjugommen, maa dette jpges pdelagt beo Mibler, ber for deme har en Gifts Egenffaber. Forjfiellige glibler har 
varet anvendt mod ๔ngoomment, og fom et af be birligite fan aubefales folgende:

Tobacco (ujaucet) ............. I Pound.

Water .................... 6 Pounds.

Oil of hartshorn..............6 Ounces.

Tobaffen foges i Bandet, indtil omtrent det Salve er tilfage, og naar bet er bleven foldt, fies Det, hoorefter man tiljotter Drjen. Efter at Re= fterne af ulben er flippet af, vabifes Dyrene to Sange med et faabant Bab, nemlig en Sang foer Uge. For ftorre Floffe trænges naturligvis mere end bet her anforte fivantum, men da maa man for= hoje Dette efter det angione Forhold. Foregaar Babjfningen on Binteren, maa Babet vore varmt. Sat, fom har varet $\mathrm{i}$ Berorelfe med Dyrene tioligere, maa rengiores, og alle Mioler til Sygonmens fremtioige Unogaaelje bennttes.

\section{Batteriot.}

Dette er ell Sygoom, fom har varet ligejaa lange fjellot fom den er huppig. Den optrader helit i Sommere med forbolosuis ftorre Regn= mængde.

Rjendetegu.

Man iporer forit en tiltagende Størrelje af \$ृo=

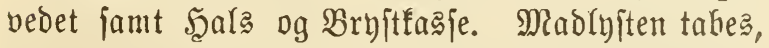
Sanigen bliver ufiffer, og tilfidit opr Doret, fom det jnes, uden Ridelfer. 


\section{$\mathfrak{U}$ a $r j a g e r u e$}

Gertil er ojtejt Bejtuing paa baarlige, vaablandte Brrasgange, eller andet baarligt Fober, rigejom ogjaa faregne Anlrg bos Dyret tan fremfarbe Syg= dommen.

$$
\text { Bella } u \text { d ri } \| \text { g. }
$$

Man maa give Inret bebre Foder on iffe udjette Det formeget for foldt oy jugtigt Bejr, famt give oet Salt og tilitreffeligt norende Foder, hoortil er jat en Sfefuld pulverijerede Juniperberries for hoert Dyr pr. Dag.

Jndovortes gives folgende fom Shedicin:

Resin, powd................. Drachms. Sulphur, powd................ Drachms. Elderflowers, powd ............ Drachms.

Mix.

En jaaban Fortion gives hoert Faar to Ssange baglig indtil Beoring indrwoer.

\section{Jgelinge i Revereu.}

Denne ejendommelige Sygoom ubmarter fig ved en feberfri Tilitano og er ofte temmelig languarig, faa ben tiot er vanjelig at opoage og beopmme. Dels angribes fun enfelte celdre Faar og dels hele Jloffe.

$$
\text { Siendetegn. }
$$

Dyrets uro begynder at falde af, faa at ber op= ftaar bare \$letter hift og her paa froppen, og iffe 
fierben plages faudaume Inr ai loft Liv og hyppig Urinladen. Slimbinderne i Maxje og Mund have et blegt eller gulagtigt lubjeende, og langere henne under Sygoommen bemarfer man vandagtige $\mathfrak{S}_{\mathfrak{a}}=$ veljer paa forifjellige Regem:dele.

\section{$\mathfrak{A}$ a r jagerne}

faut heljt joges $i$ daarlige, vaablendte og ujunde Graggange eller ell baarlig, fuap elfer uhenfigts. maşig Fooring i bet Şele taget - iffe at forglentme urent Driffebanto. Nuar et Dyr, ber bor af denne Sygoom, mlloerioges, vil mant finde, at Leverents

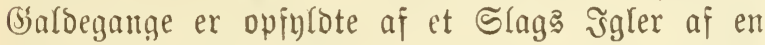
Tommes $2 a n g b e$ og af ell graagrøn Farbe og et flabtryft ubjeende. 2eberens ubjeende er forifjellig fra, huad ben er i normal Tilftand, idet ben er for= harret og instager et ftorre Dmfang end $i$ fund Tilitano.

\section{$\mathfrak{B} e \mathfrak{h a n d}$ ding.}

Dyrenes Fooring eller Brrasgang maa foranores og vige $\mathfrak{B l a d s}$ for ent mere henfigtafuarende. De maa jettes $i$ \&y under folst og regnfurdot $\mathfrak{B}$ ejr. Paa Foderet fan itroes Salt og liot pulveriferet Şarpir (resin), omtrent en Thejte pr. Dyr.

Jnovortes giver man:

Sulphate of iron ............... I Drachm. Root of angelica............... 2 Drachms. Juniperberries ............... 3 Drachms. 
En faaban ßortion gives to Siange baglig. Behandringen maa tage fin Begynderje pjeblifferig, naar man marfer Sygdommens Tiljtedevorelfe, da da ben ellers let lan antage en uthelbredelig Form.

\section{Roppendifet.}

Rjendemarferne paa Denne farlige og imitfomme Sngoom, der opftaar hurtigt og uden Forlober, er at Iyret har Feber, Mablyften tabes, Livalraften er neditemt og Aandebrattet bejucrligt, medents et

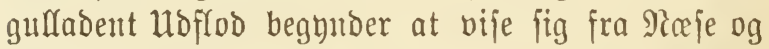
Mand. ßaa de meft blottede Steder fes rodrige, runbe finuder af en Bonnes Storrelfe, fom, maar de ffubbes bort, efterlaber en gulagtig, tyftflydende Beoffe. Forefomme de $\mathrm{i}$ ftorre Mrengde, furelte de fammen og danne fammentrengende Sforper, hoorved urben fanmenflobes i "Totter" og Dyrets Tiljtand og uldjeende banner et ubehageligt @fue.

$$
\text { ar a r a gerne }
$$

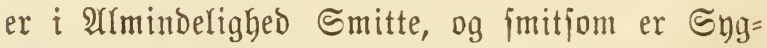
oommen i hojefte Srab og fan folge med urben, foorken benne forjendes; den fan jaalebes erfoldes ofte paa ell uffyldig og ubegribelig Maade, ja, den fan endog forplantes gjennem Ruften, bvis Syg= bommen ex herifende inden et 5 mraabe af 25 I)ile.

$$
\mathfrak{B} \text { e ha } n \text { bling. }
$$

Foderet maa være fundt og nerende, og den for= nobne Bejtyttelje mod foldt og regnfuldt Bejr maa 
ydes. De inge Dyr maa ftrengt affondres fra de funde, indtil man er fiffer paa, at Epidemien er ophort.

Snbvortes giver mant:

Glauber's-salt................. Drachms.

Root of calamus .............. Drachms.

Sulphate of iron ............. D Drachm.

Black antimony.............. I Drachm.

Mix.

En jaaban Fortion gives en Ssang Daglig indtil Beoring, og alt muligt maa giores for at fore= bygge Smitjtoffets ubbredelie.

\section{Hitopdere.}

Dette hoift bejnuberlige Tilfalde forefommer iffe fielden inden en Faareflof, idet et eller flere Dyr begnnde at ade Ulden af de purige. Man vil ret indje, at dette er en faare uheldig Dmitendighed, Der bringer $\mathfrak{T} a b$ for Fjeren, Goorfor man hurtigit mulig maa foge at forebngge dente ftngge Mani ho vebfommente Dyr.

\section{Uarjagerne}

til benne uheldige rilbøjelighed rigger ofteit $i$ en baarlig Blanding af Forbojelfesvodjferne eller Syre= bamneljer i Tarmfanalerne. Şvis Sygoommen eller Tilbpjeligheden opftaar hos dette eller bint Dinr, medens de er opitaldede, er fom oftejt Sulte= fobring Rarjagen, ibet be forjultue Dyr foger at faa 
fat $i$ Spror og Aloner, jom har hangt fig fajt $i$ de andres $\mathfrak{H}$ to. Effterabelfe er ogjaa for enfelte Dyrs Bedfommende Aarjag til denne Iilbpjelighed.

$$
\mathfrak{B} \in \mathfrak{h} a \mathfrak{b} \mathfrak{i} \mathfrak{i} \mathfrak{g} \text {. }
$$

Fr Sultefobring Yarjagen, maa man felofolge= rig give en rigerigere Fooring, jom det bebjte $\mathfrak{M i d}=$ del $i$ Dette Tilforlde. Saar man berimod udfundet, at en intre Engelighed ligger til Brrund herfor, maa denne inges fiernet ved pasjende Mioler, faajom en Blanting af:

Bi-carbonate of potash ........... 2 Drachms. Chalk.................... I Drachm. Root of gentian .............. 3 Drachms. Mix.

En jaadan Portion gives Dyret to (Sange baglig proxis, og bet bor famtioig affonores fra be porige og futu un og ba fitlles fammen med den porige flof for at faa Biaheo om, at bet har glemt fin tibligere Tilbojelighed. Shois Surent for enfelte Dyrs $\mathfrak{B} e d=$ fommende iffe faar det forwnjfede Mofald, bor ned = fommende Dyr ftrax orcbes.

\section{3enbrub.}

Untoer Foripg paa at hoppe over Gjjerder (jump fencess), eller- hoor Beitęmarfen er opfyldst af ßodber og Stubber, hander det iffe fielden, at Ben= brub finder Sted, og da dette tiọt fan gialde varbi= 
fulde Stambyr, bil Behandingen lonne fig, medens millore varbifulde Dyr bedjt brabes, jo for, jo heller.

Behand $\mathfrak{i}$ il g.

Dyret affondres fra be purige og gives et $\Re$ um med jeunt Bsulv. Man underiøger Benffaden møj= agtig og ioger at ubfinde Bruboets Bejfaffented. Benftubberne fitilles mu líge mob Ginanden, og itarf Eobife paabades Stedet, der da ombindes med en 4-6 Tommer bred Irerredsftrimle. Derefter laver man fig en anden lignende Lerrebsjtrimle, ber paa den elle Side jmores goot ind med Bsibs (Plaster of Paris) omtrent faa tyf fom ent tyno (jrob. Dette lagges rundt Benbrubdet og ombitides med Iraade, faa at bet fitboer tret ind til Bentet. Naar bette tor= res, dauner det et Sanliter, Der hindrer Benemberne fra at foranore Den rigtige Stilling.

\section{Faareblinbbed.}

Dentue er en epibemiff Sygdom, fom optrader periodevis og inden visfe Tiosrum. Det fines fom

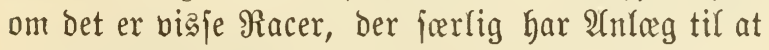
fange Sygdommen, men atter til enffelte Tidoer gaar den ogjaa udenfor dente Regel. Dent er i bojejte Brad jmitfom og angriber neften uden Undagelfe huert enejte Dyr i groffen.

\section{Rjendetegn.}

Dyrente innes at beffytte $\$ D$ junene faa meget fom muligt nod Sollyfets anobirfninger, og under $\mathfrak{B} e=$ 
ftrabeljen paa at beffntte dem render de mod enfuer Sjjenftand, der ligger $\mathrm{i}$ deres $\mathfrak{B}$ j. Dj. Dinene ere rin= dende og itcerft betcendte og ved normere Refigtelie vifer bet jig, at J్ornhinden er forịtorret og har an= taget en fra det normale afvigende Farbe.

$\mathfrak{A}$ arjager.

Den vigtigite er Smitte, ibet ๔ygdommen over= fores fra den ente Faareflof til den anden. Jels tan ogjaa Brinoheden opitaa ved, at Dyrene under $\mathfrak{B i n}=$

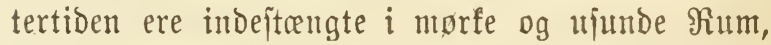
hoor Ammoniafounjter fammen med Morfet frem= falber jamme.

\section{Be $\mathrm{g}$ a $n$ o ling.}

Er bet muligt, bor Dyrente fodres under et Sfyggebrot (shade), jom Solitraalerne iffe fan trange igjentem, eller paa jfyggefulde Browgange, hoor ber er riçelig anleoning til at erfolde foldt, jrift $\mathfrak{B a n d}$. Fr det nogle af Faarene, fom enonu iffe er angrebne of Sygoommen, bor bisje burtigit muligt affonores fra be angrebne.

Paa \$Djente antvender man polgerde Middel:

Sulphate of quicksilver .........2 Drachms. Tincture of opium............ D Drachms. Rainwater .................. Ounces.

Mix.

Tre til=fire Draaber Geraf oryppes ind i 2 jet to Sange daglig. Unagen hermed vil rigelig lonne fig, thi benne Sngoom bringer ofte Dorene til en 
mager og afflappet Tilftand, der foraarjager $\mathfrak{T} a \mathfrak{b}$ jaavel af $\Re$ joo fom Ulo. Er bet varmt Bejr, faut man en Gang baglig overiprojte Dyrentes Spoveder med $\mathfrak{B}$ and om $\mathfrak{A}$ nlediting gives.

\section{Evage 3er.}

Denne Sngoom opitaar pludjelig uden Forlober, ibet juart et, fnart et andet Dyr i Floffen angribes. Den er iffe fmitfom, men ben er meget generende for Dyrente, ijor naar be befinder fig paa Ssxasgangen, idet be iffe har Beorgeliesenne not til at foge om= fring efter Fode.

$$
\mathfrak{A} \text { a } \mathfrak{j} \text { a gerne. }
$$

Den vigtigite $\mathfrak{A}$ arjag er ell baarlig Fooring eller baarlige, vandinge Srrasgange, hois Srrosarter iffe indefolde de møovendige Ralfitoffe til Benbygnin= gens Styrfe. Dels fan Aarjagen rigge $i$ en altfor ftcri Fooring med ftorft feotbannento Fooerftoffe, hoorved Benente faar en forbget, uforholdsmasfig Bregt at brere, men byppigit er ong Aarjagerne be for nownte.

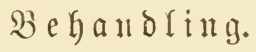

Fr det muligt, bor man af og til lade Dyrene faa frifft Rlopergres, men bet maa fremfor alt vare goot. S̄notraffer Sygonmmen $i$ Sommertioen, og Srrasgangens Beffaffented er, fom ovenfor nabnt, bor Dyrene, om muligt, henjottes paa en bebre Srrasgant, helft en hojtliggende. 
Intonortes giver man ftroet paa Foberet eller $i$ Driflenandet:

Chalk ................... Io Ounces.

Magnesia.................... Ounces.

Root of calamus................. Ounces.

Mix.

En fulo Epifejte for Goert Faar itroes paa Foder eller i Driffeuano, fom noevnt, to Santge baglig. Saajnart man morfer, at Den rette Bevageljesevne

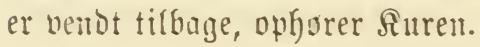

\section{Bejuerlige foifler.}

\section{Torfosicrue fommer, men iffe sovebet.}

is bette Tilfalde fees forfooderne i Fobjel: aabningen, men Şovedet fan, trobs Dyrets Beer, iffe jees. Man maa ba befrygte, at Det enten maa norre trangt til Siben eller truffet nebab moder Bryjtasien. I begge Tilforlde er Fobjel paa na= turlig slaabe umulig. Det forite, her bor giores, er at finbe en jmaffer Sine, bvis begge Fnder juo= res.om Bienene, hoorpan be puttes ind igjen, hoor= ned man faar beore $\Re u m$ til at arbejoe for at finbe

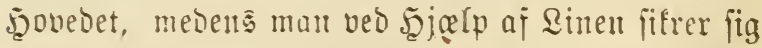
Benene, medens dette joregar. Med en med Jedt 
indjmurt Sุaand underiøger man ફूovedets Stilling, og er man paa bet Rene hermed, indforer man ent Tang, fom ftadig burbe haves og fer faaledes no:

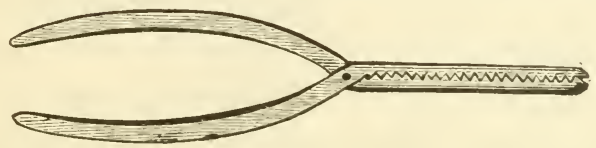

Man foger un at faa fat $i$ Foiterets ene Dre og traffer ved dette Tag Sovedet fremad og itrammer fantioig i Rinerne, der ere fritede til Benene. Treffente forfigtigt i Rinerne og Tangen famtibig med yldoderoyrets $\mathfrak{B e e r}$, vil Fodjelen let foregaa.

\section{5ovedet vifes, men iffe Benene.}

Şovebet er her rigtignof for fit Bebfommende $i$ Drben, men Benenes ฐgjenbliven Ginorer Fobjelen, og Sixlp tiltronges.

Man tager et Snore og friter oet rundt Foite= rets Underficue, huorpaa bet fores tilbage til Rejet og Fobjelsoejene er frie. Mes Tangen foger man III at rette Benene, oer $i$ Urmindelighed ligger ind under Foiterets $\mathfrak{B u g}$; fieldnere indager de en anden Stilling i dette Tilforlde. Er det Inffedes at faa Benene frem i Fobjelsaabningen, troffer man lem= peligt under $\mathfrak{B e e n e}$ i Linerne og Fobjelen foregaar med $\Omega_{t}$ thed. Fofterets Underfice maa juarejt mulig befries for Rinien og Stedet babes med foldt Band. 


\section{3uglauds ซึodiel.}

Denne unaturlige Stilling hiororer ofte fra ben Dmitandighed, at Fofteret er blevet forjfubt under itærfe Beer, og det er ofteft $\mathfrak{T} i l f x l b e t$ med ftarfe liväraftige Foitere. Seer fan der iffe vare Tale om at giore Forjog med at vende Fofteret, men bet maa fobes i benne Stilling. Rommer begge Bagbentene tilinne $i$ Fobjelsaabningen, bor nan berfor iffe for foge at traffe $i$ dem, thi Fodjelen vil $i$ hoj Grad vanjfeliggiores, hois iffe Salen famtioig folger disje. Shafen rigger fom ofteft $i$ Bugt oppe under Modor= Dyrets Sryds, og med Tangen maa man til at faa fat i Şaleñ Fndefpios og traffe Denne fremover $i$ Retning med Benene. SRu vil Fobjelen foregaa ganje med famme Lethed, fom om den havde waret naturlig.

\section{Toiteret Dobt i MEoder Riv.}

Er Fofteret Doot i Moders Riv, vil altio Fod Fe= fen blive bejocerlig og neppe ffe uben funtig Sूjolp, felo om fopiteret indtager et rigtigt Leje; thi i Dette Tilferbe juulmer Fofteret op til en uforbolosmesfig Storrelie, Beerne ere ofteit ophorte og Fobjelavejen tor og blottet for de flimede Foitervadjer, ber i $\mathfrak{A l}=$ mindelighed. Yetteliggion de levende Foiteres Foojler.

Da Foiteret allerebe befinder fig $i$ en halu for raabneljestiljtano, giolder bet om at faa et $\mathfrak{T} a g$ Foiteret, jom fan holde. Man naa i paafommende 
Tilfwlde vare forinnet med en Rrog af folgende $\mathfrak{U} d=$ feento med tilfaitet Rine:

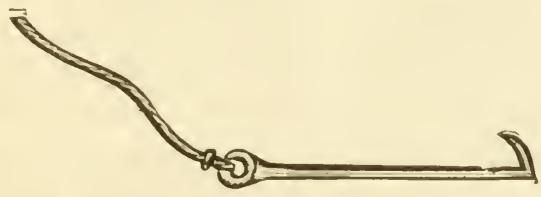

Denne Srog foges fajtet $i$ et af Djenfulferme, thi det er det fiffefte Sted, fom i dette Tilforlde fan vaelges, idet Sirogen da faar fæite $i$ felve Bande= bentet. Fobjelsuejen maa indjmores godt med Drje, og er Rrogen fátet paa fit Sted, fan man lempelig treffe $i$ benue, famtioig fom man maa have $D p=$ merfjomheden henventot paa, at Benene er i Droen. Bed Forjigtighed fra Foojelahjalperens Side vil det i Arrmindelighed lyffes at faa frem $\mathfrak{F}$ ojteret og redoe Dyrets Liv.

Banffelige Fopdfer fan ofte oræebe Moderoyret, og da bette tiot fan variere $i$ en $\mathfrak{B} a r b i$ fra fem til ti Dollars, figer bet fig felv, at ben $\mathfrak{U}$ mage, mant har hermed, vil lonme fig, naar Dyret freljes. Bed Dvelfe opnaar man juart at bline gode Frobjels=

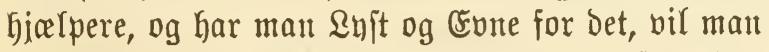
juart funne yoe Goiffetjombeljt Şusbyr ben formodne

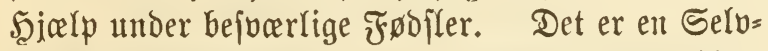
folge, at Moderbyret er bet vigtigite at faa rebdet, medens Fojteret er underorbuet, men er man heldig nof at redoe begge, jaa meget dejto bebre.

om ₹aareracen. 3. 


\section{Betaubelie i Fobjelsweien.}

Betandelfe opltaar tiot efter bejocrlige Fodjler, og er en Sygoom, der fræver jerlig Dpmarfjombed. Ser man, at Dyret vifer Uro, ftamper $i$ Jorden, fajter fig ned og ftaar op, famt ubvifer paafaldende Sraftloghed over Rrybjet; at Dyret har en dinglende Sang $i$ Forbinderje med Feber, jamt trænger paa jom under Fodjelen,- - da er det uben Toivl angrebet af bemte Sygonn.

\section{$\mathfrak{B}$ e $\mathfrak{h}$ a $\mathfrak{u}$ d $\mathfrak{i} \mathfrak{n g}$.}

Man maa give Dyret god ftyrfende Sliobriffe, Geljt beftanende af Scauremel, om det haves for haanden. Ino i Fodjelsvejente fprojtes en Blanding af lige Dele $\mathfrak{B a n d}$ og Edoife.

Sndvortes anvender man:

Saltpetre ................ 2 Drachms. Glauber's-salt ............. 4 Drachms. Root of valerian ............. I Drachm.

Mix.

En jaaban ßortion gives to Ssange daglig.

Dyret maa vogtes vel for Irrefvind og alt, fom fan fremfalde- Fortiglerfe.

\section{Qumuefaituing.}

Denne Spgoom er periobiff, om man faa vil faldé det, "idet den fun i enferte $\mathfrak{A}$ ar med fortere eller rængere Mellemrum indfinder fig. Sygdommen er oprindelig iffe jmitiom, men fan tilflut optrade 
med en Smittejngons ufravifelige Rjendetegn. Det figer fig jelv, at Sygoommen er forbundet med ftorre eller minore $\mathfrak{T a b}$ for Ejeren - alt efter dens uditrefning. Saajnart et Faar har faftet Fofteret, det vere fig ude eller $i$ Şus, maa Det affondres fra de porige, og alt, hyad Der ftaar $i$ Forbullolje med bet fajtede Fofter, faaveljom Fofteret jelv, maa bje= brifferigfiernes. Moderbyret maa gives goot og nærende Foder og vogtes for Forfiolerfe.

Med Senjun til de obrige Dyr, der cuomu iffe har vift Tegn til হammefaftuing, maa man ind= ffrenfe fig til at forebygge den faa meget jom mu= ligt. De maa faa en god Fooring, rent Driffebanto og iffe nojottes for foldt og regnfuldt Bejr. \$aa Foderet fan ftroes en Blanding af folgende:

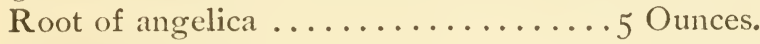
Root of gentian.............. 5 Ounces. Bark of oak .................. Mix.

Af denne Blanding ftroes en Spifejfefuld for Gvert Donr paa Foderet en Bang Daglig $i$ en til to Uger, idet man da fan forudjotte, at ber er ingen Fare mere.

\section{Efterbyrben bliver tilbage.}

Efter Rammefajtning vil (5fterbyrben ofteft ban= ifelig løanes af fig felv, hoorfor Doret befinder fig i en libende Tilftand. Ser er iffe Tale om at funne

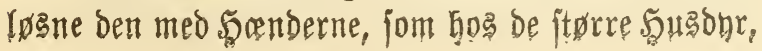


ba man bertil mangler $\Re u m$ nof, hoorfor man ved Sjiclp af Miediciner, ber udeluffende virfer i benne

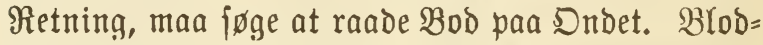
forgiftning eller $i$ heldigite Fald Mfrugtbarked fan blive Folgen af enl Efterbyrbe, der gaar over $i$ Selp= fortæring. Man giver Dyret forgende Blanding: Ergot of rye ................ D Drachms. Juniperberries ................ D Drachms. Mix.

En jaaban Portion gives 2 Sange baglig, indtil Efferbyrden løänes, hyilfet ofteft ffer inden et $\mathfrak{T} i d s=$ rum af otte Dage. 


\section{Dm Slagtnina.}

Det er iffe alene $\mathrm{i}$ levende Rive, at \$ूusnyrente

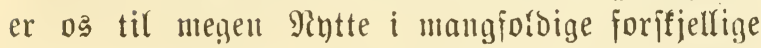
Retninger--hvert efter fin Bejtemmelie, men endog efter Dpoen tjener de Mrenneffene til Babn, ibet deres $\Omega$ iød tiener og til Fode, og deres Scud eller Sfind, Ben, Scaar, Scorn ofp. forarbejoes til many= folbige nuttige og unnboerlige Ssjenftande. Şvis de altio opde en naturlig Dob, funde intet vajent= rigt vare at fige herom, men Sagen er, at Mienteffet uben videre gaar hen og berøver dem Livet, naur og hoor bet finder for godt, Hoen andre \$ूeninn end at giore fig ben Benntterfe af ben, fom de forbaanden= varende Dmitandigheder forbrer.

Bed at vere fig benne Rjendsgienning beviojt, huor ftor Sppororing matte ber iffe vare til at berøve den Rivet paa den mindit fmertefulde og. Dyret meft ubevidite Maade! Scer er et af de rigejte Felter for menneffelig Medfolelje, men paa fanme Tib, hoor ofte tiljidefattes iffe disfe Seninn og maa

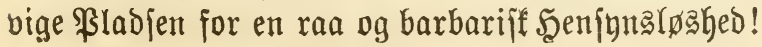


Bi har ßligter mod Dyrene, medens de lever og er os anbetroede til Forjorg, men disje \$ligter ophorer fandelig iffe $i$ det $D_{j}$ jeblif, vi ftaar $i$ Begreb med at

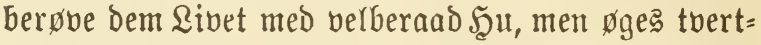
inod til Da at bevife dem vor fiofte $\mathfrak{B l i g t}$ og Mied= libenked bed at give Dem en imertefri og jaaviot muligt ubevidit Dod. Bi har Lov til at brabe de Dyr, hyis Dod er os til Sytte eller deres \&iv er til Sfabe for ${ }^{2}$, men det er underforitaaet, at vi er pligtige til at labe Deres ufrivillige Affgang fra $\mathfrak{B e r}=$ den ffe paa en Maade, ber er overengftemmende med Menueffelighed og Rrijtelighed.

Da ber jo paa enfoer Farm foregaar Slagt= ninger, og ba be bedite, mejt imertefrie og hurtigfte Maaber fauffe for Manges Bedfommende endnu iffe er jaa armindelig fjendte, ffal jeg $i$ det \&iterfolgende fremptille de for de forjfjellige Şusour fifrefte og bedite Slagtningsmaaber.

\section{seiten.}

Seftens Slagtning bor beitaa enten $i$ Cfyoning efler ved et belrettet Slag $i$ Panden. It flaa ben for \$anden,forbrer \$Delie, og fun øbede Folf bor - foretagé det; thi et Fejljlag giør Duret faa Defperat, at bet jenere ofte er temmelig vanffeligt at giore oet af med bet. Forend man giver det afgiorende Slag, giør man nogle "blinde" Slag, fom man falder, ibet Dren eller Slağgen fanfes og baves nogle Sange 
mod det betegnede Sted, forend Şovedflaget fores. 5erved vinter man mere Sifferhed og Driftighed; fremfor alt maa Sibbegynderen iffe undlabe at be= nutte fig af Denne \$overje.

Med Sূentinn til Stedet, hoor Slaget bor traffe, bemarfes, at det iffe bor falde midt $i$ ßanden, men narmere et af \$Djuene, da \$andebenet er tyffere $i$ Midten end normere $\$ D$ juene. Seejteris Dojne maa altio $i$ Forvejen tildoffes, faa ber iffe yives ben nogen Foreftilling om den fficbnejuangre Stund, den gaar imøde.

Enfelte pbede Slagtere bruger ogjaa at bibringe Seiten et Rnivitif $i$ Raffen, huorved Rygmarven eller den forlangede Şjerte overifjares og Dyret ftyrter bevioftlos om jom truffet af et $\mathbb{2} n$ n. Hovede Folf bor bog iffe foretage fig Denne Slagtning maabe, da den frover abjtillig Doerje, men om Nogen ffulde øujfe at lare den, er bet bedit at praftifere med en bod Şejt, ber $i$ Forbejen oog iffe maa vare ftuffen $\mathrm{i}$ Naffen. Man lagger ba en Snor eller lignende rundt Dyrets Şals, faaledes at den netop berorer $\$$ Drernes bagerite Rand. Miot mellem begge \$Drer og heldente Rniven libt til fig forer man benne med et rajft Støo ned mellem Scalahvirvlerne der, foor Snoren ffjorer Şalafammen. Stedet er mellent anden og trebie \$ृalæhvirbel, og træffer man rigtigt, innfer Iniven, Der heljt bør vare toeagget, fra fire til fem Tommer onfot, men ftober man fejl, 
gaar ben fun ubetydeligt $\mathfrak{i}$ Dybden, og ftopper $\mathfrak{i}$

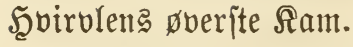

Naffejtifningen er en ret og jmertefri Dobs= maabe, men ben forbrer jom jagt \$Doelje for at fumne udfores med Sifferked.

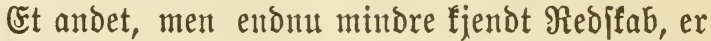
Slagtentajten. Dent er meget henfigtsmexsfig og Gurbe haves paa enfyer Farm, da ben fan bruges

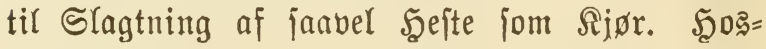
jtaaende Tegning vifer omtrent Denz Udjeende, naar Den f. Ex. ligger ubitraft paa et Borb.

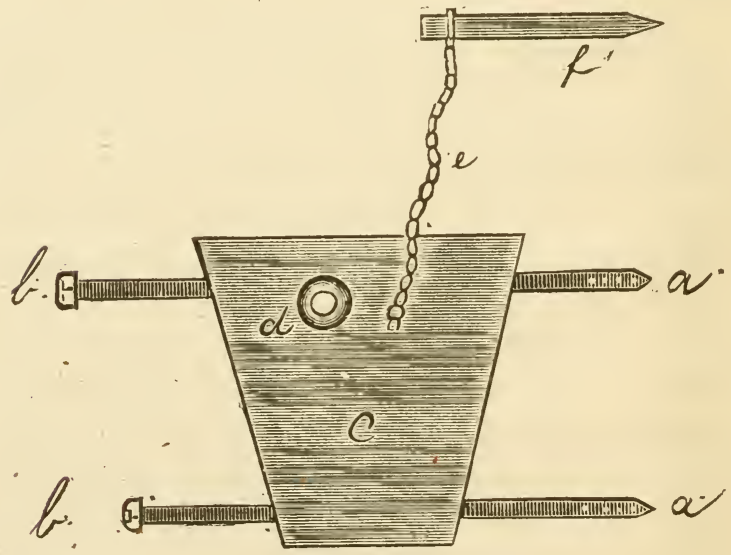

Bogitav c beteguer felve Maften, der giores af almindelig Sfolwder. Sed Şixlp af Remmerne a-a og b-b jpendes Maffen fajt til Dyrets ßande, idet de jattez-en lavere og en trojere oppe - under 
Dyrets Rjouver; d er en Cylinder, ber er indinet i Majfen, og fom naa have en \&nngde af en Tomme. Denne rager ub fra Maffen og ex beftemt til at holde f, der er ell tre Iommer lang, ffarp Staalpios, fom med Rjeden e forbiudes med Maffen. Maar demte er rigtig paapandt, ffal den boffe Djnene, medens Cylinderen maa traffe ret foran $\mathfrak{B} a n d e n$ mellem Djuene, og narmere et af Disfe. Sku tager man Staalpibjen, foutter den ind i sylimberen, og med en almindelig Snedferhammer gives den et fraftigt Slag, hoorbed den trenger gjennem Sjjerneffallen ind i Sejernen, og Duret ligger fulditandig bevioft= los for Slagterens Fobder.

Slagtemaffen har bet ftore Fortrin, at endog Uøvede fan med Sifferbed bruge dell, og der fau intet uhyggeligt Dptriu forefalde ved deng Brug.

Bil man ffyde Sceften, maa bette Gelit ffe ved Şixlp af en Riffer, hyis en faadan haves ved ફূaan= Den. For at vare fiffer paa at traffe det Sted, man

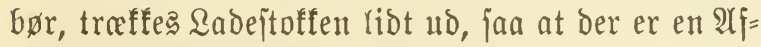
ftand mellem Siffelpibens Munding og Sadeftoffens Ende af omtrent otte til ti Tommer. Den fioft= naunte rettes nu fagte og ftille len paa det beteg= nebe Sted, og Suglen vil folge Den angivne Retning.

\section{So e II.}

Roens Slagtning ffer i Armindeligbed ved Slag for ßanden eller i Raffen, eller ogfaa bed det an= gaaende Sef̣tens Slagtning omtalte ?affeftif. 
Med Şeninn til Slag for ßanden giwlder det ogjaa her, at der forores Sovelje for iffe at foraarjage Forityrrelje $i$ Slagtningen og unøbvendige Libeljer for Dyret. Man maa, fom ovenfor omtalt, poe fig i blinde Slag, forend man ubforer bet virfelige Slag, da man derved erfolder mere Sifferfed og Rolbblodighed.

Enfelte foretreffer at rette Slaget mod ఇaffe= grubent, og for dent Hovede er bette Gellere at fore= traffe, fuut at det er fraftigt nof. Bed den fterfe Scienteryftelfe, fom derved opftaar, falder Dyret be= viditlos om.

Raffeftif fan med ftorre Rethed udfores paa

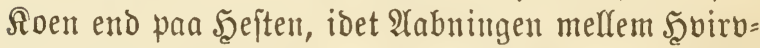
lerne hos den forite er ftørre, ment Fremgangänaaden med at finde dente er netop den famme fom hoe Sheiten. Jeg vil jarrig anbejale Denne fiofte Maade for Roens Bedfommende, huig iffe Slagtemaffen fjendes, thi om man end itroer fejl, faa vir Rniven

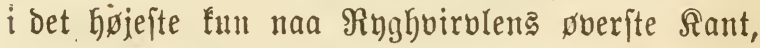
huilfet iffe giør Duret farbeles umedgiørligt. For at faa Blobet til at lobe tro, girres et Stif indad og

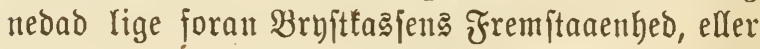
mant fan flaa ud $\mathcal{L} u f t=o g$ Spiferpr jaameget, at mant ab ben $\mathfrak{B} e$ fan blotfitille Blobaarerne og berefter overifjure bem.

Den famme Maade til Blodudtapningen an= vendes ogfaa for Šeftens Bedfommende. 


\section{Fra $\mathfrak{a} \mathbf{x} \mathfrak{e}$.}

Med Senfyn til bette Slagtning bemorfes, at man bor bedpoe det forft ved Sjjelp af en alminoelig Scammer, idet det gives et fraftigt Slag lige i $\mathfrak{B a n}=$ Den. Derpaa folger Stifningen, der iffe, fom En= felte giør, behøuer at utofore paa ben Maade, at man flaar Saljen furdjtandig op. Man griber med Den venitre நaand blot om \&uft og Spiferdret, tvinger disfe faa godt fom nuligt ub fra Şalahvirb=

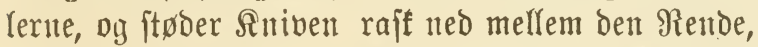
jom herved dannes. Fr Rniven ffarp og iffe altfor breb, vil bet nof lobe heldigt af.

\section{Svinet.}

Rigeoverfor Svinet maa enten Slag eller Sfyd= nintg blive be almindeligite Slagtemaaber.

Sfyoning for Stifuingen maa anjes fom lettejt og Gurtigit. Mange bruge hertillanos at tage fat paa Dyret, oftejt to til tre Maxnd, flaa det ned og Derpaa itiffe det, hoorefter det raver rumbt indtil bet oververloes af Doben. Sitedetfor dette burde man for it lade det faa en Revolverfugle i Şiernen. Det er bedft at rabe Sfubdet gaa ind f. Ex. lige bag hrire $\$$ Dre og i Retning mod venftre $\$$ je. En Mand fan mu magelig ftiffe bet alente, og al Sfrig og Larm unbgaas.

2Ul Slagtning bør foregaa med $\Re$ og Sindig= hed. En Slagtning fan ofte rent uo bribe grufom 
ved at man taber ฐindsligebagten. Dm man end ex ndojaget til at orabe et Dyr, der har varet til Sfade, da bor dog Bredell mod det tabe fig $\mathrm{i}$ Dobs= pjebliffet, jaa at man iffe paaforer Dyret unpoven= Dige Sibeljer. Man maa erindre, at Dyr er Dyr, og at de blot forger fit naturlige Jultinft, og iffe, fom Menneffet, fan Danne fig Fornnftilutninger om

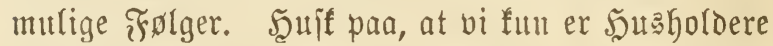
over anbetroet (3odz, og at Regnffabzongens Romme tun er et Tidspporgamaal. 

Rueft: Tnrlagebog om Seften, ned 5. (S) altung, norit=ameritanjét Drrla et Billebe.

Indbuttoen ..........................

Emnft inibunben $i$ Gloth ..............

Seiteventelt. - Sgandobog for Farm $\mathfrak{A f}$ ફ. (B)altung.

LIBRARY OF CONGRESS

InbGunden

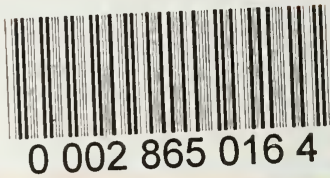

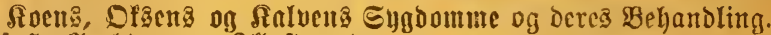

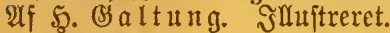

Inobutben

256 ta.

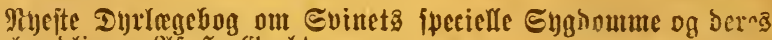
Behanding. $\mathfrak{U} \mathfrak{f} \mathfrak{S}$. Blaltung.

Intbundoen.

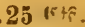

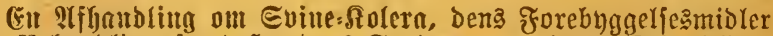
ag Behandling, famt fyutben Engboume og Deres ibehandlitg. थी $\mathfrak{g}$. (B) altung.

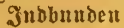
.25 (5)

Dim Fanreracer famt Faarenez faregne Sngdomme og beres

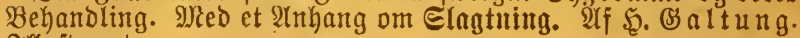
Эlluptreret.

Indbunden

.25 (5t8.

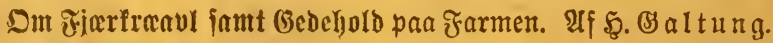
Indbutuben .........................................25 5 tธ.

Dim Melfens Befandling og Emortilvirfntug. Tilegnet

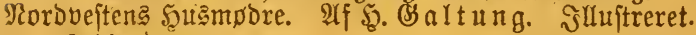
9ndbutber ...........................................25 (6t8.

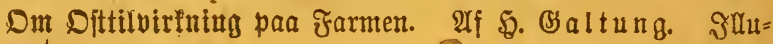
itreret.

Inbbunben .23 (5).

Farmeren Beileder i Durlegemedienen. Beffribelfe ober 100 af De vigtigfte Dyrmediciner, Deres Birtning, Forefomft, Samm ticetning, Dofiz óg 2lituentelfe $i$ alle foretommende Sns

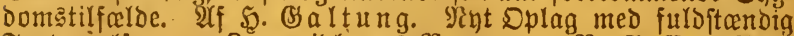

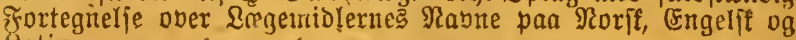
Satin.

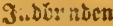

$.25(5 \pm 8$

Proresie: SPandin wen's Boghandel,

183, $185 \& 18 \%$ Rorth Reoria Et., Chicago, gur. 